\title{
The first evaluation of brain shift during functional neurosurgery by deformation field analysis
}

\author{
D Winkler, M Tittgemeyer, J Schwarz, C Preul, K Strecker, J Meixensberger
}

J Neurol Neurosurg Psychiatry 2005;76:1 161-1 163. doi: 10.1136/jnnp.2004.047373

Stereotactic surgery is based on a high degree of accuracy in defining and localising intracranial targets and placing surgical tools. Brain shift can influence its accuracy significantly. Deep brain stimulation of the subthalamic nucleus can markedly change the quality of life of patients with advanced Parkinson's disease, but the outcome depends on the quality of electrode placement. A patient is reported in whom the placement of the second electrode was not successful. Deformation field analysis of pre- and postoperative three dimensional magnetic resonance images showed an intraoperative brain movement of $2 \mathrm{~mm}$ in the region of the subthalamic nucleus (the target point). Electrode repositioning resulted in efficient stimulation effects. This case report shows the need to reduce risk factors for intraoperative brain movement and demonstrates the ability of deformation field analysis to quantify this complication.

$\mathrm{S}$ ereotactic functional neurosurgery is a well established and accurate method and offers increasing possibilities for treatment. ${ }^{1-3}$ Currently, stereotactic localisation based on computed tomography (CT) and magnetic resonance imaging (MRI) data is the most common method of guiding the neurosurgeon during functional neurosurgery, an accuracy of less than $1 \mathrm{~mm}$ being achieved with the use of high precision stereotactic frames. ${ }^{45}$ Nevertheless, the accuracy of "static" image guided devices decreases with the length of surgery owing to intraoperative brain shift. ${ }^{6}$ Methods for quantifying this brain movement (teleradiography, pointer based measurement of brain shift, intraoperative ultrasound) do exist, but are not suitable for evaluating the shift from the surface to the middle of the brain during functional neurosurgery. Only intraoperative CT or MRI can give detailed information on intraoperative shift that can be used to modify the planned trajectories. This requires that one of these examinations is repeated during surgery, with the patient still fixed in the stereotactic frame. Up to now, illustrative models for shift measurement in the brain, especially in the basal ganglia, have not been established. In this study we undertook a vectorgraphic brain shift analysis in a patient in whom electrode displacement occurred because of shift of central brain structures.

\section{CASE REPORT}

The patient, who was 70 years old and suffering from Parkinson's disease, developed severe motor fluctuations, dyskinesia, and gait disturbance. Because dopaminergic drug treatment had resulted in the development of psychotic symptoms, the therapeutic possibilities were limited. Treatment with levodopa $950 \mathrm{mg} / \mathrm{d}$, a COMT inhibitor $400 \mathrm{mg} / \mathrm{d}$, and pergolide $1 \mathrm{mg} / \mathrm{d}$ failed to achieve satisfactory relief of symptoms. On these grounds the patient qualified for a trial of deep brain stimulation (DBS). Preoperatively, the unified Parkinson's disease rating scale (UPDRS) part III was applied and the score improved from 48 to 20 at 45 minutes after an oral dose of $200 \mathrm{mg}$ levodopa.

\section{Image acquisition, planning procedure, and functional neurosurgery}

The imaging protocol included multiplanar Tl weighted (three dimensional magnetisation prepared rapid gradient echo, time of repetition $(\mathrm{TR})=10 \mathrm{~ms}$ ) and $\mathrm{T} 2$ weighted (inversion recovery fast spin echo, time of echo $(\mathrm{TE})=3500 \mathrm{~ms}, \mathrm{TR}=30 \mathrm{~ms}$ ) sequences (matrix $256 \times 256$, slice thickness $1 \mathrm{~mm})$. Contrast media $(0.1 \mathrm{mmol} / \mathrm{kg}$ body weight Gd-DTPA, Magnevist, Schering, Germany) was given to visualise neighbouring blood vessels. The subthalamic nucleus (STN) was identified by Tl and T2 weighted image fusion, using the RSPS planning workstation (Stryker, Howmedica, Leibinger, Germany). In this patient, who had normal configuration of the ventricles, the distance between the AC-PC midline and the target was $10.6 \mathrm{~mm}$ on the right and $11.0 \mathrm{~mm}$ on the left. The AP position of the right sided target was $3.5 \mathrm{~mm}$ behind and $3.2 \mathrm{~mm}$ below the midcommissural point; on the left side, the respective positions were $3.7 \mathrm{~mm}$ and $3.3 \mathrm{~mm}$.

For the operation, the patient lay supine with the back and head raised to $45^{\circ}$. The left sided electrode was inserted stereotactically (ZD, Stryker, Howmedica, Leibinger, Germany) through a frontal hole under mild sedation. Multichannel microelectrode recording with five trajectories was carried out (Leadpoint 4, Medtronic, Minneapolis, Minnesota, USA) and provided the typical functional signature of the target in four of the five trajectories. Before final electrode positioning the target was confirmed by the best patient response to stimuli using macroelectrodes. With stimulus parameters of frequency $130 \mathrm{~Hz}$, current $2.0 \mathrm{~mA}$, and pulse width $60 \mu \mathrm{s}$, suppression of the tremor was achieved without any side effects.

The procedure was repeated on the opposite side 220 minutes after the start of the surgery. Unfortunately no typical STN signal could be found in any of the five trajectories. Test stimulation led to a left sided hemiparesis and abducens paresis of the left eye, associated with muscle twitch and paraesthesiae in the ipsilateral extremities. We did not observe tonic contraction of the contralateral extremities, which would be expected if stimulation was affecting the pyramidal tract. Stimulation was varied from 1.0 to $6.0 \mathrm{~mA}$ ( $130 \mathrm{~Hz}, 60 \mu$ s pulse width) without improved clinical effect. A permanent electrode was placed and its position identified by postoperative MRI, using the same technique as for the initial surgical planning. Because image based confirmation of electrode position is not routinely done under stereotactic conditions, the postoperative MRI was carried out after removal of the stereotactic frame. Picture analysis showed that the final position of the electrode was displaced from the planned target coordinates by $2 \mathrm{~mm}$, probably because of a surgery associated brain shift. This accounted for the absence 


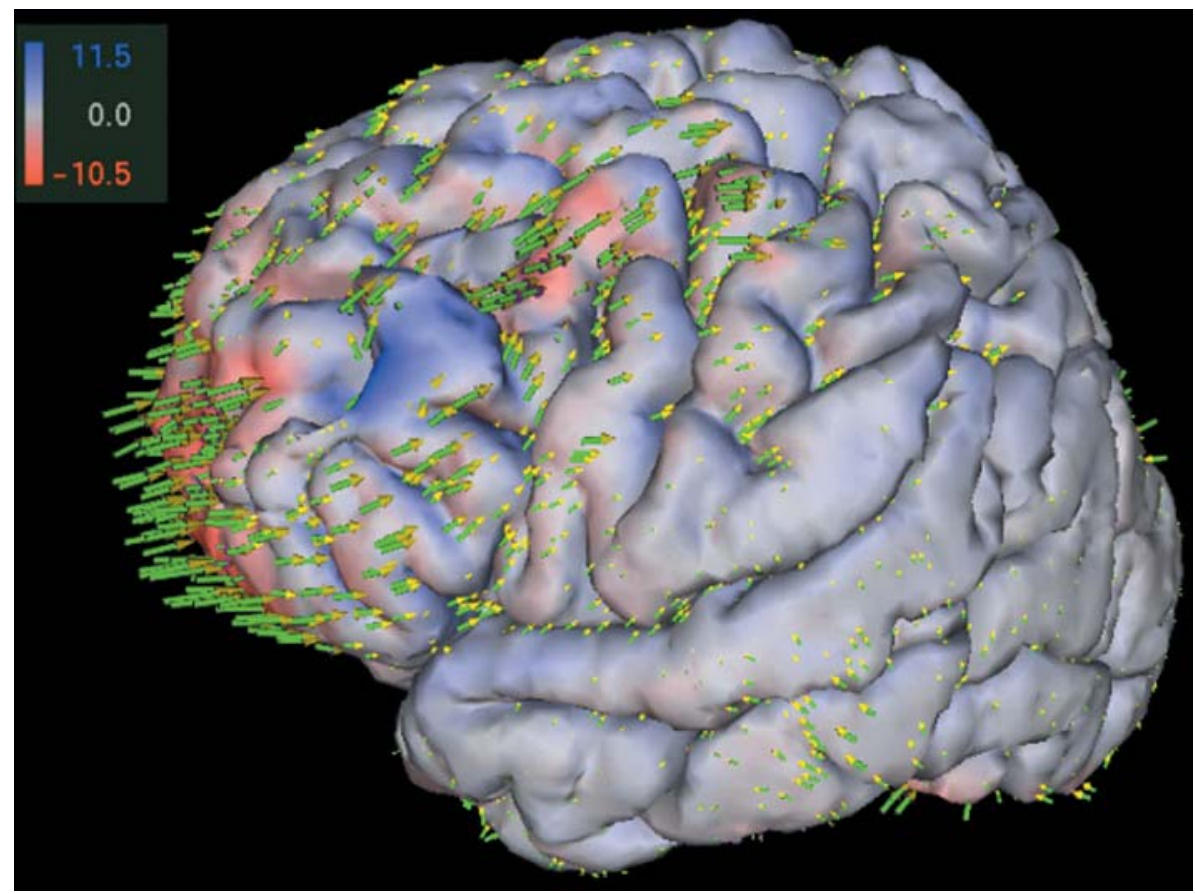

Figure 1 Lateral surface depiction of the patient's brain. The brain shift is indicated by the yellow tipped green arrows, which show the direction and extent (arrow length) of brain displacement. Note that shiffing occurs at the brain surface and also in deep brain structures (see fig 2).

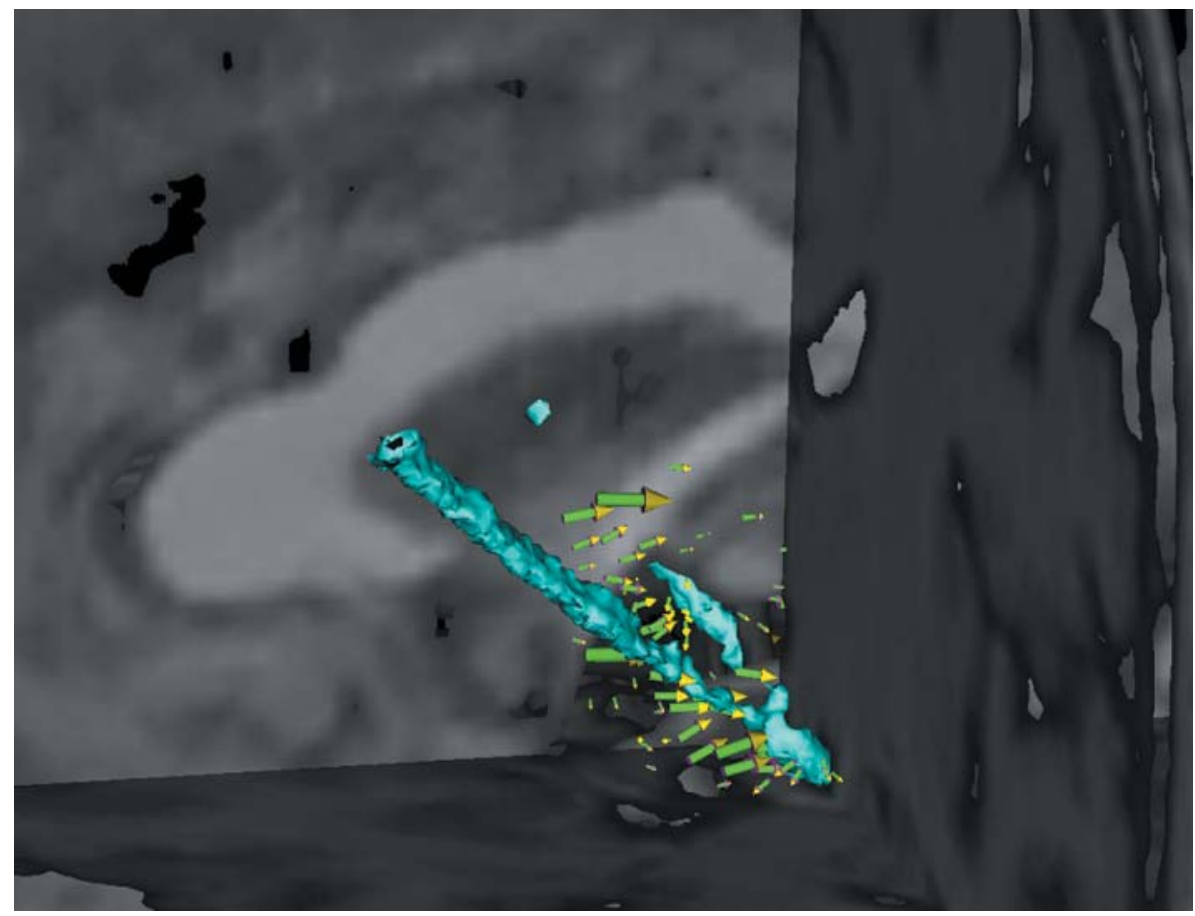

Figure 2 The admission channel to the subthalamic nucleus is shown in turquoise. Brain shift in the target region is indicated by the yellow tipped green arrows, which show the direction and extent (arrow length) of brain displacement. For clearer visualisation this region has been cut from the overall displacement field. The strongest shift appears in the circumference of the admission channel, though a prominent amount of shift is also present in structures that are not in direct contact with the intervention channel. This can be accounted for by brain shift. 
of the expected microrecording and macrostimulation responses.

Patient had a further operation on the next day, in which the displaced electrode was replaced using the original target coordinates. Microrecording and macrostimulation were sufficient in three of five trajectories, with no side effects. The implanted electrodes (DBS electrode, model 3389, Medtronic) were connected to a pulse generator in the left infraclavicular area (Kinetra Impulsgenerator, model 7428, Medtronic). A detailed deformation field analysis was carried out retrospectively to verify the electrode displacement (figs 1 and 2). Postoperatively, stimulation in the off medication state was associated with great improvement in tremor, rigidity, bradykinesia, gait, postural stability, and activities of daily living, with concurrent improvement in the UPDRS motor score.

\section{Deformation field analysis}

First, the MR images were preprocessed. The first process was to reorient the images to the AC-PC plane. The images were processed for grey scale normalisation and removal of intensity inhomogeneities. Rigid registration was applied in order to align objects in the images for position and orientation. To assess volumetric values for each ventricle, the ventricular system was segmented as described by Hojjatoleslami and Kruggel, ${ }^{7}$ and a non-rigid registration was employed to monitor residual differences between the images which reflect morphological (local) changes in a three dimensional deformation field. In view of the large deformations that originate from the neurosurgical approach, the non-linear registration was based on a fluid dynamic model. ${ }^{8}$ The vector field was then displayed in one to one size relation to the underlying MR image. Hence it could be directly superimposed on the image to allow direct measurement of changes, either manually or automatically. In this case a tissue shift of $13 \mathrm{~mm}$ near the brain surface and $2 \mathrm{~mm}$ in the STN could be documented, which confirmed our suspicion that a shift problem was the reason for electrode displacement.

\section{DISCUSSION}

Earlier studies were designed to evaluate the "total clinically relevant error" in placing the electrodes for deep brain stimulation, comprising errors associated with imaging, target selection, vector definition, and the mechanical errors of the different stereotactic frames. There is little knowledge about dynamic intraoperative changes in the brain. This single case study was carried out to document the brain shift as a "dynamic error" occurring during functional neurosurgery. With the help of deformation field analysis we were able to identify a mean frontal cortical brain shift of $13 \mathrm{~mm}$ and a tissue shift of approximately $2 \mathrm{~mm}$ in the region of the STN. The difference in brain shift between the cortex and the midbrain probably results from the fixation of central brain mass by bony and falcine structures.

The results of our study provide information about the degree of intraoperative brain shift during stereotactic procedures. With functional neurosurgery even minimal brain shift in the region of the STN cannot be neglected.
Brain shift as a reason for "dynamic error" is caused by loss of cerebrospinal fluid (CSF), the inherent qualities of the brain, failure to reclose the burr hole, the time consuming procedures of multichannel microelectrode recording and stimulation testing, and by the procedure itself, which favours further CSF derangement. Deformation field analysis has proved to be a reliable method for analysing intraoperative brain shift. Up to now no other study has demonstrated this phenomenon with such accuracy.

\section{Conclusions}

Our study has confirmed the brain shift associated loss of positional accuracy during functional neurosurgery, using deformation field analysis. To guarantee a safe stereotactic procedure and to prevent any undesirable side effects it is worth balancing all time consuming measures, including the electrophysiological evaluation, against possible adverse events. It is essential to minimise possible surgical risk factors and to finish the procedure promptly if there is any suspicion of electrode displacement. Because of the possibility of electrode displacement, image based confirmation of the final electrode position under stereotactic conditions is highly recommended.

\section{Authors' affiliations \\ D Winkler, J Meixensberger, Department of Neurosurgery, University of Leipzig, Leipzig, Germany \\ M Tittgemeyer, C Preul, Max-Planck-Institut for Neurocognition and Neuroscience, Leipzig, Germany \\ J Schwarz, K Strecker, Department of Neurology, University of Leipzig \\ Competing interests: none declared}

Correspondence to: Dr Dirk Winkler, Klinik für Neurochirurgie, Universität Leipzig, Liebigstr 20, 04103 Leipzig, Germany; wind@ medizin.uni-leipzig.de

Received 9 June 2004

In revised form 11 October 2004

Accepted 15 December 2004

\section{REFERENCES}

1 Limousin $P$, Krack P, Pollak $P$, et al. Electrical stimulation of the subthalamic nucleus in advanced Parkinson's disease. N Engl J Med 1998:339:1105-11.

2 Kupsch A, Earl C. Neurosurgical intervention in the treatment of idiopathic Parkinson disease: neurostimulation and neural implantation. J Mol Med 1999:77:178-84.

3 Valalik I, Sagi S, Solymosi D, et al. CT-guided unilateral thalamotomy with macroelectrode mapping for the treatment of Parkinson's disease. Acta Neurochir 2001;143:1019-30.

4 Schulder M, Fontana $\mathrm{P}$, Lavenhar MA, et al. The relationship of imaging techniques to the accuracy of frameless stereotaxy. Stereotact Funct Neurosurg 1999;72:136-41

5 Hariz MI, Bergenheim AT. A comparative study on ventriculographic and computerized tomography-guided determinations of brain targets in functional stereotaxis. J Neurosurg 1990;73:565-71.

6 Nimsky C, Ganslandt O, Cerny S, et al. Quantification of, visualization of, and compensation for brain shift using intraoperative magnetic resonance imaging. Neurosurgery 2000:47:1070-80.

7 Hoijatoleslami SA, Kruggel F. Segmentation of large brain lesions. IEEE Trans Med Imaging $2001 ; 20: 666-9$

8 Wollny G, Kruggel F. Computational cost of non-rigid registration algorithms based on fluid dynamics. IEEE Trans Med Imaging 2002;21:946-52. 